\title{
Systematic Review \\ Texture-Modified Diets, Nutritional Status and Mealtime Satisfaction: A Systematic Review
}

\author{
Xiaojing Sharon $\mathrm{Wu}^{1, * \mathbb{C}}$, Anna Miles ${ }^{2}$ and Andrea J. Braakhuis ${ }^{1}(\mathbb{D}$ \\ 1 Faculty of Medical and Health Sciences, Discipline of Nutrition, The University of Auckland, \\ Auckland 1010, New Zealand; a.braakhuis@auckland.ac.nz \\ 2 Faculty of Science, School of Psychology, Speech Science, The University of Auckland, \\ Auckland 1010, New Zealand; a.miles@auckland.ac.nz \\ * Correspondence: xiaojing.wu@auckland.ac.nz
}

Citation: Wu, X.S.; Miles, A.; Braakhuis, A.J. Texture-Modified Diets, Nutritional Status and Mealtime Satisfaction: A Systematic Review. Healthcare 2021, 9, 624 https://doi.org/10.3390/ healthcare 9060624

Academic Editors

Daniela Figueiredo and Oscar Ribeiro

Received: 10 May 2021

Accepted: 21 May 2021

Published: 24 May 2021

Publisher's Note: MDPI stays neutral with regard to jurisdictional claims in published maps and institutional affiliations.

Copyright: (c) 2021 by the authors. Licensee MDPI, Basel, Switzerland. This article is an open access article distributed under the terms and conditions of the Creative Commons Attribution (CC BY) license (https:// creativecommons.org/licenses/by/ $4.0 /)$.

\begin{abstract}
While the association between dysphagia and malnutrition is well established, there is a lack of clarity regarding the nutritional status and mealtime satisfaction of those consuming texture-modified diets (TMDs). This systematic review summarises and critically appraises the nutritional status and mealtime satisfaction of adults consuming TMDs. A systematic database search following PICO criteria was conducted using Cochrane Central (via Ovid), MEDLINE, CINAHL, EMBASE and Scopus. Nutritional status, mealtime satisfaction and costs were identified as primary outcomes. Eligible studies were grouped according to outcome measurement. In total, 26 studies met the inclusion criteria. Twenty studies evaluated the nutritional status by weight change or using malnutrition screening tools and found the consumption of TMDs correlated with weight loss or malnutrition. Nine studies evaluated mealtime satisfaction, with two reporting poor satisfaction for people on thickened fluids (TFs). Nutrition intervention through adjusting texture and consistency and nutrition enrichment showed positive effects on weight and mealtime satisfaction. The majority of the studies were rated as 'neutral' quality due to the limited number of experiments. TMD consumers had compromised nutritional status and poor mealtime satisfaction. More research input is required to identify promising strategies for improving the nutritional status and mealtime satisfaction of this population. Food services need to consider texture, consistency and fortification in designing menus for people on TMDs to avoid weight loss and malnutrition, and to enhance mealtime enjoyment.
\end{abstract}

Keywords: texture-modified diet; dysphagia; swallowing impairments; older adults; malnutrition; mealtime satisfaction

\section{Introduction}

Dysphagia has been defined as a difficulty in swallowing food or drink safely and efficiently, and can occur anywhere between the oral cavity to the stomach [1]. Approximately $8 \%$ of the global population are reported to suffer from dysphagia, and in the developed world, the prevalence appears to be increasing alongside the ageing population [2]. Neurological disease is a common cause of dysphagia, including acute stroke, Parkinson's disease and dementia. Dysphagia can lead to severe consequences, including choking, aspiration, pneumonia and increased risk of mortality [1,3].

A high prevalence of weight loss and malnutrition has been reported in people with dysphagia, accompanied by nutrient deficiencies [4-6]. The co-existence of dysphagia and malnutrition has been reported in acute and long-term care (LTC) settings, with a higher prevalence of malnutrition for those with dysphagia [7-9]. Supporting this link, previous research has identified dysphagia as a decisive risk factor for malnutrition, and conducting nutritional and clinical interventions appears to reduce the incidence of malnutrition among dysphagic populations [10-13]. 
An essential and effective current clinical nutrition intervention for those with dysphagia is diet modification using prescribed texture-modified diets (TMDs), including thickened fluids (TFs) $[3,11,14]$. Standard TMDs are commonly softened, chopped, and minced or blended into the recommended texture and consistency by adding water.

Patients consuming TMDs may have negative perceptions of their meal experience. Poor compliance with dietary recommendations has been reported [15-17]. Possible causes include reduced mealtime pleasure from altered and restricted consistencies, prolonged mealtimes, swallowing exhaustion, physical difficulties in tolerating large fluid and food volumes, dependency of feeding, and associated cognitive impairments [18-20]. In addition, it is recognised that TMDs can be inherently less nutrient-dense due to the additional liquid requirements for processing [10]. This leads to an increased risk of compromised nutritional status [21-23].

Interventions have begun to be explored to optimise nutritional intake in order to lower the risk of unintentional weight loss and malnutrition. Some published reviews have indicated that nutritional supplements, meal fortification with nutrient-dense ingredients, energy-dense meals and offering nutritious in-between meals are considered as favourable interventions for malnutrition [24,25]. Other interventions include modifying meal contents or aesthetics by adjusting flavour, texture and consistency, and reforming the food appearance.

The association between TMDs, mealtime satisfaction and malnutrition warrants consideration by foodservice providers and carers who need to provide safe, nutritious and aesthetically appealing food to at-risk populations. The provision of food plays an essential role in health outcomes and quality of life for older adults [3]. Even in the context of a single meal, the ability to improve meal consumption and enjoyment exist. For example, offering variety within a meal increases consumption [26]. Several reviews have addressed TMDs in relation to (1) the swallowing safety of those with dysphagia [27-29], (2) the use of TMDs in specific populations [18,30], (3) quality of life [31] and (4) prevalence of dysphagia [5]. Therefore, while the safety and prevalence aspects of dysphagia have received research attention, the nutritional implications and mealtime satisfaction of TMDs have not been so thoroughly summarised and analysed. In a previous systematic review, we investigated nutritional intake in those on texture-modified diets [32]. The purpose of this paper was to systematically identify and examine the available evidence on nutritional status and mealtime satisfaction of adults using TMDs and TFs. The research questions were: what is the nutritional status of TMD consumers, and are they satisfied with the current TMDs?

\section{Materials and Methods}

This systematic review followed the PRISMA-P reporting checklist. The protocol was registered on PROSPERO CRD42019134897.

\subsection{Selection Criteria}

The PICO framework was used to assess study eligibility. Eligible study participants were prescribed any level of TMDs or TFs, and aged 18 years old. The adult age bracket was chosen to maximise data collection and study inclusion. Studies were excluded if participants were on a clear fluid diet, which is commonly prescribed to patients with gastrointestinal disease to minimise digestion rather than being an intervention diet for swallowing difficulties [33]. Studies were considered eligible if they assessed any of the following clinical measurements: anthropometry comparison or changes, nutritional status referring to the incidence of malnutrition, mealtime satisfaction and financial meal cost. Studies that reported the prevalence of malnutrition but did not include assessment details were excluded. Mealtime satisfaction, including commentary and/or ratings of TMDs or TFs, could be reported by either patients or staff. Experimental, observational, cohort and cross-sectional designs were included. Studies were excluded if they were case studies, reviews, expert opinions, conference paper, uncompleted clinical trials or non-English publications. 


\subsection{Data Sources}

This systematic review was conducted alongside our previous review assessing the nutrition intake and meal content of TMDs in May 2019 [32]. The authors used the same database (Cochrane Central (via Ovid), MEDLINE, EMBASE, SCOPUS and CINAHL), and searching strategies, and updated the search in April 2021 (see Supplementary Data S1 for search strategy). With assistance from a senior librarian, search strategies were developed based on the various descriptions of TMDs and TFs.

\subsection{Data Collection and Analysis}

All search results were imported to Mendeley Desktop 1.19.4 (Mendeley, London, UK) [34]. One author screened titles and abstracts following PICO criteria. If the author considered the study eligible, a full-text article was retrieved and reviewed by two authors for final inclusion. The reference lists of full-text articles were manually screened for additional relevant studies. One author performed data extraction, crosschecked by the second author using a structured data collection form developed in Microsoft Excel for Office 365, version 1902 (Microsoft Corporation, Albuquerque, NM, USA) [35]. Data extracted included authors, year, settings, country of origin, study design, sample size, mean age, aetiologies, TMD/TF levels, interventions, nutritional status and mealtime satisfaction outcomes and measurement tools, key findings and limitations.

Eligible studies were categorised and summarised based on the outcome measurement (weight, nutrition status, mealtime satisfaction and cost). Observational studies and experimental studies were analysed individually under each outcome. Where three or more studies described the same intervention and outcome measurements, a meta-analysis was conducted.

The Academy of Nutrition and Dietetics Quality Criteria Checklist (QCC) for primary research was used for study quality assessment, including the risk of bias. Studies were graded as positive if issues of criteria, bias, generalisability, data collection and analysis were clearly addressed. Studies that were neither exceptionally strong nor exceptionally weak was graded as neutral. Negative studies indicate that not all issues have been adequately addressed.

\section{Results}

Following the PICO criteria, 26 eligible studies were included for final analysis, including thirteen observational studies, nine experimental studies and four RCTs. Title and abstract screening were conducted for 5239 non-duplicated studies. Fifty-eight articles were then chosen for full-text assessment, with an additional nine studies manually identified from reference lists (see Supplementary Data S2 for PRISMA chart). Two types of intervention were used to improve TMD quality. Firstly, to improve food appearance, shaping, moulding, texture/consistency modification were applied. Secondly, optimisation of the nutritional content was achieved through fortification or adding extra supplements.

\subsection{Nutritional Status}

Participant characteristics and study outcomes are presented in Table 1 . Studies were conducted across 14 countries and various settings including hospitals $(n=11)$, LTCs (long-term cares) $(n=11)$, a combination of both $(n=2)$ or within the community $(n=2)$. Of the 26 studies, 19 were published after 2010. All 11 studies that assessed nutritional status used the MNA (Mini Nutrition Assessment) or MNA-SF (Mini Nutrition Assessment-Short Form) and were published in the last ten years. The age of the 7428 participants (1165 and 5863 from experimental and observational studies, respectively) ranged from 59 to 105 years old.

Though pureed food was the most common TMD studied, terminologies and descriptions of TMDs varied across countries. Types of TMDs/TFs included International Dysphagia Diet Standardisation Initiative (IDDSI) levels, UK descriptors (texture B, C, D, E), Japanese seven stages of TMDs, liquidized, mashed, minced, minced/pureed, chopped, soft 
and bite-sized, easy mastication and blended. TFs were labelled as honey-, nectar-, puddinglike, mildly thick and moderately thick viscosity, and Stage 1-3 from UK descriptors.

A total of 20 studies included measurements of body weight (BW), Body Mass Index (BMI), handgrip strength or malnutrition screening. Eleven observational studies assessed the nutritional status between TMD and standard diet consumers, as shown in Table 2.

A correlation between malnutrition or significant weight loss and consumption of traditional TMDs was found in all studies, with two exceptions $[17,36]$. On the other hand, using modified TMDs as an intervention showed positive weight changes compared to the traditional TMDs, found in all experimental studies (Table 3). Reyes-Torres et al. found handgrip strength was also significantly improved by consuming 12 weeks of consistency-modified TMDs [37]. The prevalence of malnutrition in patients consuming traditional TMDs ranged from $18.4 \%$ to $59 \%$. MNA scores less than 17 or MNA-SF score less than 8 were classified as indicating malnutrition [38-40]. Zanini et al. reported the indicator of risk of malnutrition significantly reduced after 6 months of personalised textured TMDs $(p<0.001)$ despite scores still being in the range of at risk for malnutrition $(\mathrm{MNA}-\mathrm{SF}=10)$ [41]. Similar results were also found by Martín et al., with significant improvement of the malnutrition indicator (MNA-SF $=9.84 \pm 2.05$ vs. $11.31 \pm 2.21$, $p=0.0038)$ and a lower proportion of patients who were malnourished or at risk of malnutrition ( $78 \%$ vs. $34 \%, p=0.0013)$ [42]. On hospital admission, dementia patients with dysphagia had a higher prevalence of malnutrition and a significantly higher risk of malnutrition compared to those without dysphagia [43]. Mean MNA-SF scores measured by Miles et al. and Vucea et al. also indicated the TMD consumers in LTCs were at risk of malnutrition [44,45]. Similar MNA-SF results were found in hospital patients consuming TMDs [39,46]. Additionally, hospitals offering multiple levels of TMDs ( $\geq 6$ stages of TMDs) achieved improvements in both nutritional status and swallowing abilities [39]. 
Table 1. Characteristics of included studies in the systematic review.

\begin{tabular}{|c|c|c|c|c|c|c|c|}
\hline Source & Method & Setting, Origin & $\begin{array}{c}\text { Participant } \\
\text { Characteristics }\end{array}$ & $\begin{array}{c}\text { Interventions/ } \\
\text { Intervention Period }\end{array}$ & Control & Outcomes & $\begin{array}{c}\text { Quality } \\
\text { Assessment * }\end{array}$ \\
\hline $\begin{array}{l}\text { Bannerman and } \\
\text { McDermott } \\
\text { (2011) [15] }\end{array}$ & $\begin{array}{l}\text { Observational } \\
\text { Cross-sectional }\end{array}$ & $\begin{array}{l}3 \text { LTCs } \\
\text { Scotland }\end{array}$ & $\begin{array}{l}\text { Residents }>60 \text { y } \\
\text { Ex: Nil by mouth, receiving artificial } \\
\text { nutritional support, fluid restriction, } \\
\text { acutely unwell, palliative } \\
\text { Mean age (y) } 88.1 \pm 5.4\end{array}$ & $\begin{array}{c}\text { Texture C-Thicker } \\
\text { pureed: } n=11 \\
\text { Texture } \\
\text { D—Minced/moist: } \\
n=4 \\
\text { [UK national } \\
\text { descriptors 2009] }\end{array}$ & $\begin{array}{l}\text { Standard diet } \\
\quad n=15\end{array}$ & $\begin{array}{l}\text {-Weight comparison } \\
\text {-Nutritional status }\end{array}$ & Neutral \\
\hline $\begin{array}{l}\text { Cassen et al. } \\
\text { (1996) [47] }\end{array}$ & $\begin{array}{l}\text { Pre-post } \\
\text { Experimental } \\
16 \text { days }\end{array}$ & $\begin{array}{l}\text { LTC } \\
\text { US }\end{array}$ & $\begin{array}{l}\text { All residents consumed pureed diet } \\
\text { Ex: Discharged or passed away }\end{array}$ & $\begin{array}{l}\text { 3D shaped pureed diet } \\
\quad n=18\end{array}$ & $\begin{array}{l}\text { Unshaped pureed diet } \\
\qquad n=18\end{array}$ & $\begin{array}{l}\text {-Mealtime satisfaction } \\
\text { (survey and staff report) } \\
\text {-Cost }\end{array}$ & Neutral \\
\hline $\begin{array}{l}\text { Cassen et al. } \\
\text { (1996) [47] } \\
\text { Follow-up study }\end{array}$ & $\begin{array}{l}\text { Cross-over } \\
\text { cohort } \\
12 \mathrm{~m}\end{array}$ & $\begin{array}{l}\text { LTC } \\
\text { US }\end{array}$ & $\begin{array}{l}\text { Residents consumed pureed diet for } \\
\qquad \geq 1 \mathrm{~m}\end{array}$ & $\begin{array}{c}6 \mathrm{~m} \text { of 3D shaped } \\
\text { pureed diet } \\
n=13\end{array}$ & $\begin{array}{l}\text { Unshaped pureed diet } \\
\qquad n=24\end{array}$ & -Weight change & Neutral \\
\hline $\begin{array}{l}\text { Espinosa-Val et al. } \\
\text { (2020) [43] }\end{array}$ & $\begin{array}{c}\text { Prospective } \\
\text { quasi-experimental }\end{array}$ & $\begin{array}{l}\text { Hospital } \\
\text { Spain }\end{array}$ & $\begin{array}{c}\text { Dementia patients }>18 \text { y discharged } \\
\text { from hospital } \\
\text { Mean age (y) } 84.1 \pm 7.8\end{array}$ & $\begin{array}{l}18 \mathrm{~m} \text { follow up with } \\
\text { recommendation and } \\
\text { advice provided to } \\
\text { family/caregivers }\end{array}$ & $\begin{array}{c}\text { On admission } \\
n=219 \\
\text { Standard } n=1 \\
\text { Easy mastication diet } n \\
=117 \\
\text { Blended diet } n=88 \\
\text { Mixed diet } n=13\end{array}$ & -Nutritional status & Neutral \\
\hline $\begin{array}{l}\text { Farrer et al. } \\
(2016)[48]\end{array}$ & $\begin{array}{l}\text { Pre-post } \\
\text { Experimental } \\
2 \text { weeks }\end{array}$ & Hospital, Australia & $\begin{array}{l}\text { Patients }>18 \text { y consuming pureed diet, } \\
\text { medically stable and able to } \\
\text { communicate }\end{array}$ & $\begin{array}{l}\text { Moulded pureed diet } \\
\quad \text { (Texture C) } \\
n=7\end{array}$ & $\begin{array}{l}\text { Unmoulded pureed diet } \\
\quad \text { (Texture C) } n=13\end{array}$ & $\begin{array}{l}\text {-Mealtime satisfaction } \\
\text { (survey) }\end{array}$ & Neutral \\
\hline $\begin{array}{l}\text { Garon et al. } \\
(1997)[49]\end{array}$ & $\begin{array}{l}\mathrm{RCT} \\
1 \text { year }\end{array}$ & $\begin{array}{l}\text { Hospital stroke } \\
\text { rehabilitation } \\
\text { UK }\end{array}$ & $\begin{array}{c}\text { Stroke patients with previously } \\
\text { identified thin fluid aspiration by } \\
\text { videofluoroscopy } \\
\text { Mean age (y) } 76.8 \\
\end{array}$ & $\begin{array}{c}\text { TFs }+ \text { free access of } \\
\text { water } \\
n=10\end{array}$ & $\begin{array}{l}\text { TFs only } \\
n=10\end{array}$ & $\begin{array}{l}\text {-Mealtime satisfaction } \\
\text { (survey) }\end{array}$ & Positive \\
\hline $\begin{array}{l}\text { Gellrich et al. } \\
\text { (2015) [50] }\end{array}$ & $\begin{array}{l}\text { Observational } \\
\text { Retrospective }\end{array}$ & $\begin{array}{c}38 \text { clinics } \\
\text { Germany/Austria/Switzerland }\end{array}$ & $\begin{array}{l}\text { Patients with oral cancer } \\
\qquad n=1526\end{array}$ & Liquid, mashed & Standard diet & -Weight change & Neutral \\
\hline
\end{tabular}


Table 1. Cont.

\begin{tabular}{|c|c|c|c|c|c|c|c|}
\hline Source & Method & Setting, Origin & $\begin{array}{c}\text { Participant } \\
\text { Characteristics }\end{array}$ & $\begin{array}{c}\text { Interventions/ } \\
\text { Intervention Period }\end{array}$ & Control & Outcomes & $\begin{array}{c}\text { Quality } \\
\text { Assessment * }\end{array}$ \\
\hline $\begin{array}{l}\text { Germain et al. } \\
\text { (2006) [51] }\end{array}$ & $\begin{array}{c}\text { RCT } \\
12 \text { weeks }\end{array}$ & $\begin{array}{l}\text { LTC } \\
\text { Canada }\end{array}$ & $\begin{array}{c}\text { Residents aged } 65-90 \text { y admitted } \geq 3 \mathrm{~m} \\
\text { and had }>7.5 \% \text { weight loss in the last } 3 \\
\mathrm{~m} \text { or BMI }<24 \text { with dysphagia } \\
\text { evaluated by RIC tool (Alzheimer's } n \\
=8 \text {, dementia } n=6 \text {, stroke } n=2, \\
\text { Parkinson's } n=1 \text { ) } \\
\text { Ex. Cancer, chronic intestinal disease, } \\
\text { terminally ill patients } \\
\text { Mean age (y) } 59\end{array}$ & $\begin{array}{l}\text { Shaped minced, } \\
\text { minced/pureed or } \\
\text { pureed diet and } \\
\text { consistency-controlled } \\
\text { TFs using Bostwick } \\
\text { consistometer (nectar, } \\
\text { honey, pudding) } \\
n=9\end{array}$ & $\begin{array}{l}\text { Unshaped minced-70, } \\
\text { minced-3 or pureed diet } \\
\text { and uncontrolled } \\
\text { honey-level TF } \\
\text { (consistency not } \\
\text { systematically } \\
\text { controlled) } n=8\end{array}$ & -Weight change & Neutral \\
\hline $\begin{array}{l}\text { Higashiguchi } \\
\text { (2013) [52] }\end{array}$ & $\begin{array}{l}\text { Experimental } \\
\text { Cohort } \\
7 \text { days }\end{array}$ & $\begin{array}{l}17 \text { hospitals/LTCs } \\
\text { Japan }\end{array}$ & $\begin{array}{l}\text { Inpatient and residents on TMDs with } \\
\text { inadequate consumption (stroke } \\
n=19 \text {, cancer } n=9 \text {, heart failure } n=7, \\
\text { fracture } n=5 \text {, dehydration } n=4, \\
\text { pressure ulcers, } n=3 \text {, pneumonia } n= \\
2 \text {, anaemia } n=2 \text {, COPD } n=2, \\
\text { dementia } n=2 \text {, diabetes } n=1, \\
\text { Parkinson's } n=1 \text {, other } n=17 \text {, none }\end{array}$ & & & & \\
\hline $\begin{array}{c}\text { mboxemphn }=2) \\
(\text { require total } \\
\text { meal assistance } n \\
=17, \text { partial } n=6, \\
\text { none }=34) \\
\text { Mean age }(y) 81.6 \\
\quad \pm 9.3\end{array}$ & $\begin{array}{c}3 \text { days of } \\
\text { nutrient-dense } \\
\text { (enzyme-infused) } \\
\text { TMDs nutrients } \\
\text { were not diluted, } \\
\text { and volume not } \\
\text { increased } n=57\end{array}$ & 4 days of unmodified TMDs & -Mealtime satisfaction (Survey) & Positive & & & \\
\hline $\begin{array}{l}\text { Karagiannis et al. } \\
\quad \text { (2011) [23] }\end{array}$ & $\begin{array}{l}\text { RCT } \\
8 \text { days }\end{array}$ & $\begin{array}{l}\text { Hospital subacute units } \\
\text { Australia }\end{array}$ & $\begin{array}{l}\text { Patients } \geq 18 \text { y aspirated on thin } \\
\text { liquids with prescription of modified } \\
\text { or TF diet by SLTs without chronic } \\
\text { respiratory conditions or prior } \\
\text { tracheostomy } \\
\text { Mean age (y) } 79.5\end{array}$ & $\begin{array}{l}\text { TMDs (puree; minced; } \\
\text { soft } / \text { minced) + TF } \\
\text { (honey; pudding; } \\
\text { nectar) + free access of } \\
\text { water } n=13\end{array}$ & TMDs + TF $n=5$ & $\begin{array}{l}\text {-Mealtime satisfaction } \\
\text { (survey) }\end{array}$ & Positive \\
\hline $\begin{array}{l}\text { Keller et al. } \\
\text { (2012) [53] }\end{array}$ & $\begin{array}{c}\text { Pre-post } \\
\text { Experimental } \\
9 \mathrm{~m}\end{array}$ & $\begin{array}{l}\text { Hospital and LTC } \\
\text { Canada }\end{array}$ & $\begin{array}{c}\text { All dysphagic residents fully } \\
\text { consumed pureed or minced diets } \\
\text { (stroke, Parkinson's, dementia) } \\
\text { Ex. Enteral feed } \\
\text { Facility mean age } 67 \text { and } 82 \text { y }\end{array}$ & $\begin{array}{l}6 \mathrm{~m} \text { of mix of } 61 \% \text { bulk } \\
\text { and } 39 \% \text { shaped } \\
\text { ready-to-use (reduced } \\
\text { nutrients dilution and } \\
\text { easier to chew and } \\
\text { swallow) commercial } \\
\text { TMDs } n=42\end{array}$ & $\begin{array}{l}3 \text { m of bulk commercial } \\
\text { TMDs (unshaped, } \\
\text { packaged in bulk) }\end{array}$ & -Weight change & Positive \\
\hline
\end{tabular}


Table 1. Cont.

\begin{tabular}{|c|c|c|c|c|c|c|c|}
\hline Source & Method & Setting, Origin & $\begin{array}{c}\text { Participant } \\
\text { Characteristics }\end{array}$ & $\begin{array}{c}\text { Interventions/ } \\
\text { Intervention Period }\end{array}$ & Control & Outcomes & $\begin{array}{c}\text { Quality } \\
\text { Assessment * }\end{array}$ \\
\hline $\begin{array}{l}\text { Keller and Duizer } \\
\text { (2014) [54] }\end{array}$ & $\begin{array}{l}\text { Observational } \\
\text { Interview }\end{array}$ & $\begin{array}{l}5 \text { Rehabilitation and LTCs } \\
\text { Canada }\end{array}$ & $\begin{array}{c}\text { Consumed pureed diet for } \geq 1 \text { week } \\
\text { (stroke } n=6 \text {, delirium } n=2 \text {, spinal } \\
\text { cord injury } n=1 \text {, diabetic coma } n=1 \\
\text { neck cancer } n=1 \text {, Parkinson's } n=1 \text {, } \\
\text { difficulty chewing/swallowing } n=3 \\
\text { Mean age (y) } 77.3\end{array}$ & None & $\begin{array}{c}\text { Commercial (and } \\
\text { in-house made) pureed } \\
\text { diet } \\
n=15\end{array}$ & $\begin{array}{l}\text {-Mealtime satisfaction } \\
\quad \text { (interview) }\end{array}$ & Neutral \\
\hline $\begin{array}{l}\text { Kennewell and } \\
\text { Kokkinakos } \\
\text { (2007) [55] }\end{array}$ & $\begin{array}{l}\text { Observational } \\
\text { Cross-sectional }\end{array}$ & $\begin{array}{l}2 \text { hospitals } \\
\text { Australia }\end{array}$ & Dysphagic patients & $\begin{array}{l}\text { Infant-cereal fortified } \\
\text { minced/pureed diets } \\
\qquad n=17\end{array}$ & $\begin{array}{l}\text { Unfortified } \\
\text { pureed diets }\end{array}$ & $\begin{array}{l}\text {-Mealtime satisfaction } \\
\text { (interview) } \\
\text {-Cost }\end{array}$ & Neutral \\
\hline $\begin{array}{l}\text { Konishi and } \\
\text { Kakimoto } \\
(2020)[56]\end{array}$ & $\begin{array}{l}\text { Observational } \\
\text { Cross-sectional }\end{array}$ & $\begin{array}{l}\text { LTC } \\
\text { Japan }\end{array}$ & $\begin{array}{l}\text { Older dementia residents had been } \\
\text { admitted to a LTC between 2016-2019 } \\
\text { Mean age (y) } 87.9\end{array}$ & $\begin{array}{c}\text { Soft, } n=34 \\
\text { Chopped, } n=28 \\
\text { Blended, } n=9\end{array}$ & Standard diet, $n=52$ & -Weight & Neutral \\
\hline $\begin{array}{l}\text { Maeda et al. } \\
\text { (2019) [46] }\end{array}$ & $\begin{array}{l}\text { Retrospective } \\
\text { Observational }\end{array}$ & $\begin{array}{l}\text { Hospital } \\
\text { Japan }\end{array}$ & $\begin{array}{l}\geq 65 \text { y admitted to an academic } \\
\text { hospital during 2017-2018 with } \\
\text { complete nutritional screening } \\
\text { Mean age (y) } 75.9 \pm 7.0\end{array}$ & TMDs, $n=110$ & Standard, $n=3484$ & $\begin{array}{c}\text {-Weight } \\
\text {-Nutritional status }\end{array}$ & Neutral \\
\hline $\begin{array}{l}\text { Massoulard et al. } \\
\quad(2011)[17]\end{array}$ & $\begin{array}{l}\text { Observational } \\
\text { Cross-sectional }\end{array}$ & $\begin{array}{l}4 \text { LTCs } \\
\text { France }\end{array}$ & $\begin{array}{l}\text { All residents with chewing or } \\
\text { swallowing difficulties } \\
\text { Mean age (y) } 85.8 \pm 9.3\end{array}$ & $\begin{array}{c}\text { Chopped, } n=12 \\
\text { Mixed, } n=26\end{array}$ & $\begin{array}{l}\text { Standard diet, } \\
\quad n=49\end{array}$ & $\begin{array}{l}\text {-Weight comparison } \\
\text {-Nutritional status }\end{array}$ & Neutral \\
\hline $\begin{array}{l}\text { Martín et al. } \\
(2018)[42]\end{array}$ & $\begin{array}{l}\text { Quasi-experimental } \\
6 \text { months }\end{array}$ & $\begin{array}{l}\text { Hospital } \\
\text { Spain }\end{array}$ & $\begin{array}{c}\text { Acute geriatric unit patients } \geq 70 \text { y } \\
\text { diagnosed with OD during } \\
\text { hospitalisation by nurses using } \\
\text { volume-viscosity swallow test } \\
\text { Mean age (y) } 84.6 \pm 5.5\end{array}$ & $\begin{array}{l}\text { 14-day menus of TMDs } \\
\text { (texture E or C) with } \\
\text { TFs (nectar or pudding); } \\
\text { ONS for malnourished } \\
\text { or patients at risk of } \\
\text { malnutrition; oral } \\
\text { health } \\
\text { recommendations } \\
n=62\end{array}$ & $\begin{array}{c}\text { Standard hospitalisation } \\
\text { recommendations, } \\
\text { which were not applied } \\
\text { systematically nor in a } \\
\text { standardised } \\
\text { individualised } \\
\text { application } n=124\end{array}$ & $\begin{array}{l}\text {-Weight change } \\
\text {-Nutritional status }\end{array}$ & Positive \\
\hline $\begin{array}{l}\text { Miles et al. } \\
\text { (2019) [44] }\end{array}$ & $\begin{array}{l}\text { Observational } \\
\text { Cross-sectional }\end{array}$ & $\begin{array}{l}12 \text { LTCs } \\
\text { New Zealand }\end{array}$ & $\begin{array}{c}\text { Residents consuming }>3 \text { servings } / \text { day } \\
\text { of commercial TMDs (dementia } n=37, \\
\text { cognitive impairment } n=65, \text { brain } \\
\text { injury } n=25 \text {, progressive neurological } \\
\text { disease } n=9 \text { ) } \\
\text { Mean age (y) } 85 \pm 7.7\end{array}$ & None & $\begin{array}{l}\text { Commercial fortified } \\
\text { TMDs and TFs } n=67\end{array}$ & $\begin{array}{l}\text {-Weight comparison } \\
\text {-Nutritional status } \\
\text {-Mealtime satisfaction } \\
\quad \text { (interview) }\end{array}$ & Neutral \\
\hline
\end{tabular}


Table 1. Cont.

\begin{tabular}{|c|c|c|c|c|c|c|c|}
\hline Source & Method & Setting, Origin & $\begin{array}{c}\text { Participant } \\
\text { Characteristics }\end{array}$ & $\begin{array}{c}\text { Interventions/ } \\
\text { Intervention Period }\end{array}$ & Control & Outcomes & $\begin{array}{c}\text { Quality } \\
\text { Assessment * }\end{array}$ \\
\hline $\begin{array}{l}\text { Okabe et al. } \\
(2016)[57]\end{array}$ & $\begin{array}{c}\text { Observational } \\
\text { Cohort with 1-year } \\
\text { follow up }\end{array}$ & $\begin{array}{l}2 \text { mid-sized cities } \\
\text { Japan }\end{array}$ & $\begin{array}{l}\geq 60 \text { y living at home or using in-home } \\
\text { nursing care without malnutrition }\end{array}$ & None & $\begin{array}{l}\text { Minced/pureed/mixed } \\
\qquad n=339\end{array}$ & -Nutritional status & Neutral \\
\hline $\begin{array}{l}\text { Ott et al. } \\
(2019)[58]\end{array}$ & $\begin{array}{l}\text { Pre-post } \\
\text { Experimental } \\
12 \text { weeks }\end{array}$ & 2 LTCs, Germany & $\begin{array}{c}\text { Residents diagnosed with chewing or } \\
\text { swallowing receiving TMDs regularly } \\
\text { (all participants had cognition } \\
\text { impairment) } \\
\text { Mean age (y) } 86.5 \pm 7.4\end{array}$ & $\begin{array}{l}6 \text { weeks of usual TMDs } \\
\text { (completely pureed or } \\
\text { partial soft food) } \\
n=16\end{array}$ & $\begin{array}{l}6 \text { weeks of one level of } \\
\text { reshaped TMDs and } \\
\text { enriched with } 600 \mathrm{kcal} \\
\text { energy and } 30 \mathrm{~g} \text { protein } \\
n=16\end{array}$ & $\begin{array}{c}\text {-Weight change } \\
\text {-Mealtime satisfaction } \\
\text { (interview) }\end{array}$ & Neutral \\
\hline $\begin{array}{l}\text { Reyes-Torres et al. } \\
\text { (2019) [37] }\end{array}$ & $\begin{array}{c}\text { RCT } \\
12 \text { weeks }\end{array}$ & $\begin{array}{c}\text { National Institute, } \\
\text { Brazil }\end{array}$ & $\begin{array}{c}\geq 65 \mathrm{y} \text { with a caregiver and a } \\
\text { confirmed diagnosis of oropharyngeal } \\
\text { dysphagia, and consumed TMDs and } \\
\text { TFs (evaluated by V-VST and EAT by } \\
\text { dietitians) } \\
\text { Mean age (y) } 76\end{array}$ & $\begin{array}{l}\text { Consistency-modified } \\
\text { and standardised TMDs } \\
\text { and nectar or pudding } \\
\text { level TFs (measured } \\
\text { with Brookfield } \\
\text { viscometer) } n=20\end{array}$ & $\begin{array}{l}\text { Unmodified pureed diet } \\
\text { with one viscosity of } \\
\text { TFs (consistency not } \\
\text { systematically } \\
\text { controlled) } n=20\end{array}$ & $\begin{array}{l}\text {-Weight change } \\
\text {-Handgrip } \\
\text {-Nutritional status }\end{array}$ & Positive \\
\hline $\begin{array}{l}\text { Shimizu et al. } \\
\text { (2018) [59] }\end{array}$ & $\begin{array}{l}\text { Retrospective } \\
\text { Cross-sectional }\end{array}$ & $\begin{array}{l}\text { Hospital rehabilitation } \\
\text { ward, } \\
\text { Japan }\end{array}$ & 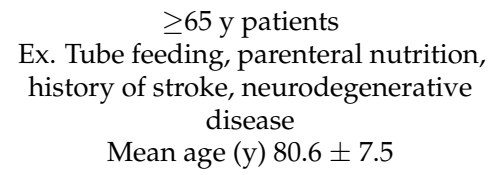 & TMDs, $n=22$ & Standard diet, $n=123$ & $\begin{array}{l}\text {-Weight } \\
\text {-Nutritional status }\end{array}$ & \\
\hline $\begin{array}{l}\text { Shimizu et al. } \\
\text { (2020) [39] }\end{array}$ & $\begin{array}{l}\text { Retrospective } \\
\text { Cohort }\end{array}$ & $\begin{array}{l}7 \text { rehabilitation facilities, } \\
\text { Japan }\end{array}$ & $\begin{array}{c}\geq 65 \mathrm{y} \text { with pneumonia enrolled in } \\
\text { rehabilitation facilities with record of } \\
\text { malnutrition screening at admission } \\
\text { and discharge } \\
\text { Mean age (y) } 82.9 \pm 9.8\end{array}$ & $\begin{array}{l}\text { Providing multiple } \\
\text { TMD stage } \geq 6 \\
n=109\end{array}$ & $\begin{array}{c}\text { Providing TMD stage }< \\
6 \\
n=109\end{array}$ & $\begin{array}{c}\text {-Weight } \\
\text {-Nutritional status } \\
\text { change }\end{array}$ & Neutral \\
\hline $\begin{array}{l}\text { Vucea et al. } \\
\text { (2019) [45] }\end{array}$ & $\begin{array}{l}\text { Observational } \\
\text { Cross-sectional }\end{array}$ & 32 LTCs, Canada & $\begin{array}{c}\text { Randomly recruited residents }>65 \mathrm{y} \\
\text { admitted for } \geq 1 \mathrm{~m} \\
\text { Mean age (y) } 86.8 \pm 7.8\end{array}$ & $\begin{array}{c}\text { TMDs } \\
\text { Bite-sized, } n=91 \\
\text { Minced, } n=139 \\
\text { Pureed, } n=68 \\
\end{array}$ & Standard diet, $n=338$ & -Nutritional status & Positive \\
\hline $\begin{array}{l}\text { Welch et al. } \\
\text { (1991) [60] }\end{array}$ & $\begin{array}{l}\text { Pre-post } \\
\text { Experimental } \\
6 \mathrm{~m}\end{array}$ & $\begin{array}{l}\text { LTC } \\
\text { US }\end{array}$ & $\begin{array}{l}\text { Residents consumed pureed diet and } \\
\text { weighed below average or serum } \\
\text { albumin/transferrin levels below } \\
\text { normal values (identified from } \\
\text { medical records) } \\
\text { Mean age (y) } 81\end{array}$ & $\begin{array}{c}\text { Pureed diets with } \\
\text { fortified high-fibre } \\
\text { cereals and commercial } \\
\text { supplements } \\
n=15\end{array}$ & $\begin{array}{l}\text { Pureed diets with } \\
\text { unfortified cereals }\end{array}$ & -Weight change & Neutral \\
\hline
\end{tabular}


Table 1. Cont.

\begin{tabular}{|c|c|c|c|c|c|c|c|}
\hline Source & Method & Setting, Origin & $\begin{array}{c}\text { Participant } \\
\text { Characteristics }\end{array}$ & $\begin{array}{c}\text { Interventions/ } \\
\text { Intervention Period }\end{array}$ & Control & Outcomes & $\begin{array}{c}\text { Quality } \\
\text { Assessment * }\end{array}$ \\
\hline $\begin{array}{l}\text { Wright et al. } \\
\text { (2005) [36] }\end{array}$ & $\begin{array}{l}\text { Observational } \\
\text { Cross-sectional }\end{array}$ & $\begin{array}{c}\text { Hospital elderly and } \\
\text { neurology wards } \\
\text { UK }\end{array}$ & $\begin{array}{c}\text { All medically stable patients } \\
\text { consumed TMDs or standard diet } \\
\text { (reasons for TMDs: } 80 \% \text { dysphagia, } \\
20 \% \text { poor dental state; stroke } n=19 \text {, } \\
\text { fall } n=8 \text {, other } n=3 \text { ) } \\
\text { Mean age (y) } 81.5\end{array}$ & $\begin{array}{c}\text { Texture B-Smooth } \\
\text { pureed, } n=10 \\
\text { Texture } \\
\text { D-Minced/mashed, } \\
n=9 \\
\text { Texture E-Soft, } n=11 \\
\text { (UK national } \\
\text { descriptors, 2002) }\end{array}$ & Standard diet, $n=25$ & -Weight comparison & Neutral \\
\hline
\end{tabular}

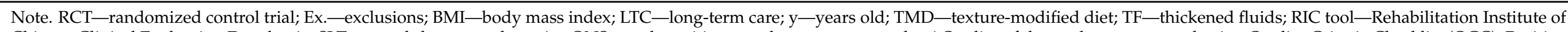

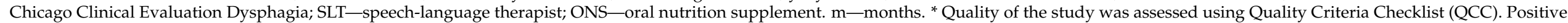

studies were identified as clearly addressed issues of criteria, bias, generalisability, data collection and analysis. Neutral studies were indicated as neither exceptionally strong nor exceptionally week.

Table 2. Outcome data for observational studies assessing nutritional status.

\begin{tabular}{|c|c|c|c|}
\hline Studies & BMI/Weight Outcomes & MNA-SF Outcomes & Nutritional Status Findings \\
\hline Bannerman and McDermott [61] & $\begin{array}{c}\text { TMDs vs. Std } \\
18.4 \pm 2.6 \text { vs. } 22.1 \pm 2.8, p=0.001\end{array}$ & & $\begin{array}{c}\text { TMDs vs. Std } \\
\text { \% Underweight }(\text { BMI < 18.5): } \\
60.0 \% \text { vs. } 6.7 \%(n=9 \text { vs. } 1)\end{array}$ \\
\hline Konishi and Kakimoto [56] & $\begin{array}{c}\text { TMDs vs. Std } \\
\text { Mild dementia: } \\
19.4 \text { vs. } 22.2, p=0.0686 \\
\text { Severe dementia: } \\
19.4 \text { vs. } 21.5, p=0.0077\end{array}$ & & \\
\hline
\end{tabular}

Oral cancer patients on liquid (61\%) and mashed (51\%)

diets were more likely to lose weight

Gellrich et al. [50] Patients who had $>10 \mathrm{~kg}$ weight loss more frequently

had to eat mashed food compared to those who had

$\leq 10 \mathrm{~kg}$ weight loss were more frequently able to eat 
Table 2. Cont.

Studies

Maeda et al. [46]

$19.0 \pm 3.8$ vs. $22.4 \pm 3.5, p<0.00$ $6.8 \pm 2.5$ vs. $11.6 \pm 2.2, p<0.001$

\section{MNA-SF Outcomes}

TMDs vs. Std
LTC residents consuming fortified TMDs Mean BMI: 23 + $5.22(13-35)$

Miles et al. [44]

\section{$\uparrow$ number of weeks on fortified}

TMDs was sig. associated with $\uparrow$ age $(p<0.05), \downarrow$ BM

or weight $(p<0.05)$ and $\uparrow$ supplement use $(p<0.001)$
MNA-SF scores were sig. correlated with BMI $(p<0.05)$ and more medical conditions $(p<0.05)$

Okabe et al. [57]

TMDs vs. Std $19.1 \pm 3.4$ vs. $20.3 \pm 3.5, p<0.001$

\section{Nutritional Status Finding}

MDs vs. Std

$\%$ Malnourished:

$62.7 \%$ vs. $6.1 \%$ ( $n=69$ vs. 213$)$

$\%$ At risk of malnutrition:

$36.4 \%$ vs. $35.3 \%$ ( $n=40$ vs. 1231$)$

$\%$ Well-nourished:

$0.9 \%$ vs. $58.6 \%$ ( $n=1$ vs. 2040$)$

TMDs vs. Std

$\%$ Malnourished:

$18.4 \%$ vs. $30.6 \%$ ( $n=7 / 38$ vs. $15 / 49), p=0.3$

$\%$ Obesity:

$31.2 \%$ vs. $38.8 \%(n=12 / 38$ vs. $19 / 49)$

\section{Fortified TMDs}

$\%$ Underweight (BMI < 18.5): 22.0\% $(n=9 / 41)$

$\%$ Overweight (BMI $>25): 29.3 \%(n=12 / 41)$

\% Malnourished: $35.5 \%(n=11 / 31)$

$\%$ At risk of malnutrition: $61.3 \%(n=19 / 31)$

$50.0 \%(n=8 / 16)$ of malnourished participant were on TMDs (MNA-SF)

$71.4 \%(n=5 / 7)$ of participants who passed away were on TMDs

Consumption of TMDs (RR: 2.93, $p=0.036$ )

and swallowing disorder (RR: $3.82, p=0.012$ )

was sig. associated with the incidence of

malnutrition and death among frail older

adults at 1-year follow-up.

TMDs vs. Std

$\%$ Malnourished:

$59.1 \%$ vs. $35.8 \%$ ( $n=13$ vs. 44$)$ $\%$ At risk of malnutrition:

$40.9 \%$ vs. $52.8 \%$ ( $n=9$ vs. 65 )

$\%$ Well-nourished:

$0 \%$ vs. $11.4 \%$ ( $n=0$ vs. 14 ) 
Table 2. Cont.

Studies

Shimizu et al. [39]

BMI/Weight Outcomes

MNA-SF Outcomes

Comparison between multiple TMDs $(n=109)$ and control $(<6$ stages of TMDs) patients $(n=109)$
At admission: MNA-SF: $8(6-10)$ vs. $8(6-10), p=0.969 ;$ BMI: $20.1 \pm 4.4$ vs. $19.8 \pm 4.4, p=0.486$

At discharge: MNA-SF: $6(4-8)$ vs. $6(3-8), p=0.459$

MNA-SF change during hospitalisation: $2.4 \pm 2.8$ vs. $0.9 \pm 3.1(p<0.001)$
TMDs vs. Std

$23.97 \pm 5.24$ vs. $26.57 \pm 5.92, p<0.001$
TMD vs. Std

$9.81 \pm 2.68$ vs. $11.37 \pm 2.13, p<0.01$

BMI and MNA-SF score the lowest in pureed

$<$ minced and moist $<$ soft and bite-sized $<$ Std.

MNA-SF was sig. negative $(\uparrow$ risk of malnutrition) associated with minced and moist and pureed diet compared to Std $(p=$ $0.03)$

Wright et al. [36]

TMDs vs. Std:

60 (39-96) vs. 62 (46-93) $\mathrm{kg}, p=0.55$

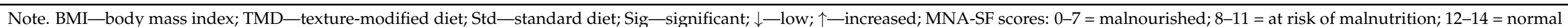
nutritional status/well-nourished. (a) Malnutrition-BMI $<21$ OR $\geq 10 \%$ weight loss in 6 months $/ 5 \%$ in $1 \mathrm{~m}$ month OR MNA $<17$ (used when MNA-SF $<11$ ); normal BMI = 21-29.9.

Table 3. Outcome data for experimental studies assessing nutritional status.

\begin{tabular}{|c|c|c|c|}
\hline Studies & BMI/Weight Outcomes & MNA-SF Outcomes & Nutritional Status Findings \\
\hline Cassen et al. [47] & $\begin{array}{l}\text { 3D moulded vs. unmoulded TMDs } \\
\text { NS weight loss in } 6 \text { months: } \\
15.4 \% \text { vs. } 100 \%(n=2 / 13 \text { vs. } 21 / 21) \\
\text { Sig. weight loss of } \geq 4.5 \mathrm{~kg} \\
0 \% 19 \%(n=0 / 13 \text { vs. } 4 / 21)\end{array}$ & & \\
\hline Espinosa-Val et al. [43] & & $\begin{array}{l}\text { Dysphagia vs. Non-dysphagia patients: } \\
7 \pm 2.68(n=211) \text { vs. } 8.2 \pm 2.45(n=35) \\
p=0.014\end{array}$ & $\begin{array}{c}\text { \% Malnourished: } 53.6 \%(n=113) \\
\% \text { At risk of malnutrition: } 43.1 \%(n=91) \\
\text { \% Well-nourished: } 3.3 \%(n=7)\end{array}$ \\
\hline Germain et al. [51] & $\begin{array}{c}\text { Shaped vs. unshaped TMDs } \\
6 \text { weeks: NS weight change }(p>0.05) \\
12 \text { weeks: Sig. } \uparrow \text { weight in shaped TMDs and weight loss } \\
\text { was seen in unshaped TMDs } \\
+3.90 \pm 2.30 \text { vs. }-0.79 \pm 4.18 \mathrm{~kg}, p<0.05 \\
\text { BMI } \uparrow \text { from } 22.4 \pm 3.93 \rightarrow 24.5 \pm 4.14\end{array}$ & & \\
\hline
\end{tabular}


Table 3. Cont.

Studies

Keller et al. [53]

Reyes-Torres et al. [37]

Welch et al. [60] BMI/Weight Outcomes

$74 \%$ of participants consuming mix of

shaped ready-to-use TMDs and bulk TMDs

achieved weight goal after 6 months

NS weight or morbidity change between intervention and

$$
\text { control periods }
$$

Trends towards $\uparrow$ weight on mixed TMDs (OR $=3.5, p=$

$0.16)$ and $\downarrow$ weight on bulk TMDs (OR $=4.3, p=0.11)$

Standardised vs. non-standardised TMDs

BMI within normal range at both admission and 6-month

$$
\text { follow up }
$$

Changes in 6 months intervention $27.76 \pm 4.42$ vs. $28.52 \pm 4.39, p=0.2045$
Changes in 6 months intervention $9.84 \pm 2.05$ vs. $11.31+2.21, p=0.0038$

Changes in 6 months intervention

$$
\% \text { Malnourished: }
$$

$18.75 \%$ vs. $3.13 \%$ ( $n=6$ vs. 1 )

$\%$ At risk of malnutrition: $59.38 \%$ vs. $31.25 \%(n=19$ vs. 10$)$

$\%$ Well-nourished patients

$21.87 \%$ vs. $65.63 \%$ ( $n=7$ vs. 17$)$ $p=0.0013$

Weight change during 6 weeks traditional TMDs: 59.3 vs $58.8 \mathrm{~kg}(-0.5 \mathrm{~kg}), p=0.21$

Weight change during 6 weeks enriched and shaped TMDs: 59.6 vs. $58.8(+0.8 \mathrm{~kg}) \mathrm{kg}, p=0.007$

Baseline MNA-SF

\% Malnourished: $25 \%((n=4 / 16)$ $\%$ At risk of malnutrition: $75 \%(n=12 / 16)$

Weight change after 12 weeks consistency modified TMDs:

$$
56 \pm 10 \text { vs. } 60 \pm 10(+7 \%) \mathrm{kg}, p<0.001
$$

Handgrip strength: $18 \pm 11$ vs. $21 \pm 13 \mathrm{~kg}, p=0.004$

NS Weight/BMI changes in traditional TMDs control group $(p>0.05)$

Weight change after 3 months vs. 6 months of fortified TMDs and supplements

$+2.8 \pm 1.25$ vs. $+4.6 \pm 2.0 \mathrm{lbs}, p<0.04$

$66.7 \% \uparrow 0.5-5.4 \mathrm{~kg}$ from $3-6$ month; $33.3 \% \downarrow 0.5-2.3 \mathrm{~kg}$

Changes after 6 months personalised TMDs

$17.88 \pm 3.48$ to $19.00 \pm 3.32(+1.12), p<0.001$ with sig. growth trend $(p<0.007)$

Zanini et al. [41]

Changes after 6 months traditional TMDs control group

Changes after 6 months personalised TMD

8 to $10(+2), p<0.001$

Changes after 6 months traditional TMDs

control group

$20.96 \pm 4.07$ vs. $17.88 \pm 3.48(-3.08), p<0.001$

Baseline MNA

\% Malnourished: 50\% $(n=20 / 40)$

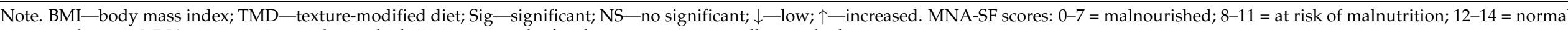
nutritional status. MNA scores: $0-16=$ malnourished; $17-23.5=$ at risk of malnutrition; $24-30=$ well-nourished. 


\subsection{Mealtime Satisfaction}

The use of an interview was the most common method for exploring mealtime satisfaction $[44,54,55,58]$. Three studies evaluated mealtime satisfaction for shaped/moulded TMDs by survey $[5,47,48]$. Keller and Duizer found that despite LTC consumers appreciating the necessity for TMDs and staff efforts, several issues contributed to the poor acceptance of pureed meals, including presentation, taste, smell, inconsistency in production, delivery and lack of variety [54].

Zanini et al. used the Edinburgh Feeding Evaluation in Dementia (EdFED) scale, which observed participants' adverse behaviours while being fed [41]. A significant improvement in eating behaviour was found with adjusted TMDs by the texture-individualised intervention $(6.65 \pm 3.13$ vs. $7.98 \pm 3.65, p<0.001)$, which was demonstrated by a high level of compliance to meal consumption [41]. Results from studies using moulded pureed foods were inconsistent. No significant differences were found in the 14-day hospital trial with moulded pureed food and 3-day trial of enzyme-infused shaped TMDs with regards to taste, appetite, presentation, ease of ingestion and overall liking $(p>0.05)[48,52]$. Nevertheless, health professionals reported shaped TMDs to be significantly more appealing ( $4.70 \mathrm{vs}$. $4.22, p<0.01)$, with a significantly higher score in joy of eating (4.44 vs. $4.11, p<0.001)$ and overall satisfaction ( 4.38 vs. $4.19, p<0.05)$ after observing patient consumption [52]. Positive feedback from staff and LTC residents was also obtained with a 16-day 3D pureed food trial [47].

Miles et al. reported that LTC residents were satisfied with the appearance and flavour of commercial fortified TMDs, with the highest satisfaction with puddings and soups (8-10 out of 10) [40]. Dryness of the meals was reported by hospital patients consuming infant cereal fortified TMDs [55].

Ott et al. reported mixed feedback from nursing staff regarding enriched and shaped TMDs [58]. Improvement of chewing or swallowing $(n=5 / 16)$, enhanced appetite and pleasure with eating was observed in some participants $(n=5 / 16)$, while one participant did not like the taste. Concerningly, 33\% of the residents $(n=5 / 15)$ were also rated by nursing staff as not receiving their prescribed diet consistency [58].

Garon et al. and Karagiannis et al. evaluated mealtime satisfaction of TFs and the alternative intervention of free water access using a survey [23,49]. Patients with access to water had a significantly higher level of satisfaction with drinks, level of thirst and mouth cleanliness $(p<0.001)$, but no significant differences in the overall feelings [23]. Garon et al. found similar results, with high satisfaction with access to water [49]. Moreover, $90 \%$ of patients on TFs $(n=9 / 10)$ reported a desire for water due to thirst, and that TFs were not thirst-quenching and lacked taste and enjoyment. Only one person in the study was satisfied with TFs.

\subsection{Cost}

The cost of TMD provision was only discussed in two studies [47,55]. Using infant cereal as fortification was 6.9 times cheaper compared to commercially fortified thickener in Australia ( $\$ 0.235$ vs. $\$ 1.61$ AUD) [55]. Cassen et al. suggested that using enhancer and thickener in 3D moulded foods could be cost-effective in increasing food intake to prevent weight loss and reduce the use of supplements and treatments [47].

\subsection{Quality Assessment}

Overall, $65 \%$ ( $n=17)$ of the studies were rated as 'neutral' quality due to the absence of follow-up. Despite 12 studies being experimental studies, only eight were rated as 'positive' quality. The other four experimental studies were rated as 'neutral' quality, mainly due to the small number of subjects and a lack of specification of drop-outs. Sample size largely varied, from 15 to 3594 [50,54]. All studies had evenly distributed numbers of participants between study groups. Results of nutritional status were collected using validated tools. Evaluation of mealtime satisfaction varied across studies depending on the questions and survey they used, as well as the cognition status of the study group. Meta-analysis was not 
possible due to the heterogeneity of study methods and settings and a small number of studies measuring the same outcomes.

\section{Discussion}

This systematic review evaluated the existing evidence of impact of TMDs and TFs on nutritional status and mealtime satisfaction. Compromised nutritional status was identified in TMD consumers. Modifying TMDs by shaping and nutrition enrichment showed promising results in improving nutritional status and mealtime satisfaction.

\subsection{Measuring Nutritional Status}

A previous literature review studied the prevalence of malnutrition in LTCs, indicating malnutrition risk is 1.7 times higher in LTC residents consuming TMDs compared with those on standard diets [5,62]. A finding mimicked in the current review. Malnutrition was reported in 33-50\% of dysphagic residents in LTCs and approximately $60 \%$ in hospital $[37,44,46,59]$.

Besides the high prevalence of malnutrition in dysphagia patients, compared to standard diet patients, those consuming TMDs were found to have poor long-term clinical outcomes, such as respiratory infection, decreased skeletal muscle mass, longer hospital stays and higher mortality rate $[39,43,46,59]$. However, the association between malnutrition, dysphagia and mortality does require further clarification. Ten studies used MNA-SF, and one used MNA for examining malnutrition. Although MNA-SF is validated for determining nutritional status in the LTC population, the inclusion of functional, psychological, and cognitive status may result in overestimation of malnutrition risk ratings. There was a lack of screening and assessment of dysphagia and malnutrition [63,64]. Only Zanini et al. and Martín et al. compared the malnutrition screening score before and after intervention and found improvement, whereas other studies only did the screening at baseline [41,42].

Weight loss was more significant in oral cancer patients on TMDs compared to those on standard diets in a retrospective study [50]. Missing documentation or measurement of weight and height was observed in LTCs, suggesting inadequate monitoring [44]. The risk of malnutrition is highly associated with weight and BMI, and as such regular assessment and documentation are necessary. A recent review suggested using the Global Leadership Initiative on Malnutrition (GLIM) criteria to assess the nutritional status of dysphagic adult patients. This covers BMI, nutritional screening tools, anthropometric measurements, body composition and dietary assessment [65]. Standardised nutritional assessment, nutrition recommendations for texture and nutrients should be included as part of dysphagia diagnosis and treatment plan $[63,64,66]$.

\subsection{Improving Nutritional Status}

Martín et al. demonstrated that the introduction of TMDs in combination with the use of high-protein, high-energy foods and an oral health intervention could significantly improve both functional and nutritional status in hospital patients with dysphagia [42]. Of eight intervention studies, significant weight or BMI improvements were found in three modified TMDs intervention studies $[37,41,51]$. Inadequate dietary intake may be contributing to the high prevalence of undernutrition [15]. The impact of nutrient enrichment on weight was only investigated in one study that reported a significant weight improvement in a LTC using a pureed diet with supplements and fibre-fortified cereals [60]. Results from previous research studying standard diets suggest food fortification improves energy and protein intake, but not body composition or functional status in nursing home participants at nutritional risk [67]. Similar results were found by Miles and colleagues, with $97 \%$ of the participants on fortified TMDs at risk of malnutrition at the time of investigation [44]. However, as an observational study, it is not possible to determine whether the outcomes were coincidental. Though nutritional status was found to be routinely screened in nursing homes, diet quality is one of the biggest challenges of clinical management which can result in malnutrition $[68,69]$. The previous review summarised that the positive improvement in nutrition intake was found using fortification and shaped TMDs [32]. Implementing 
nutrition enrichment strategies such as fortified food or supplements should be considered in dysphagic management for malnourished patients and those who are at risk of malnutrition $[68,70]$.

\subsection{Mealtime Satisfaction}

Improved energy and protein intake were achieved by optimising the texture modification $[32,71]$. The quality of TMDs is highly related to mealtime acceptance and compliance. Therefore, to guarantee adequate intake, studying mealtime satisfaction is crucial. While studies did focus on both the aesthetics and safety of the food, there was a lack of standardisation in the evaluation of mealtime satisfaction of TMDs and TFs. Poor satisfaction with TFs was found in hospital patients, resulting in poor compliance and consumption [23,49,72]. Moreover, 3D printing and shaping using silicone moulds have the potential to achieve more attractive TMDs with enhanced sensory characteristics and consistency [73]. Though no significant results were reported by patients directly, improvements were observed by staff and health professionals with the use of shaping/moulding [47,52]. Dysphagic patients require greater attention and encouragement on the acceptance of the texture modifications. Targeting additional feeding support to high-risk patients may improve nutritional status and outcome [74,75]. Clinical staff should collaborate with food service to improve meal presentation and aesthetics, as well as raising concerns regarding the appropriate texture and consistency necessary for safe swallowing. Responses using subjective measurement may be limited due to common cognitive challenges in this population. Studies may consider using observations or audits to assess mealtime satisfaction [23].

Ballesteros et al. suggested nutrient-dense commercial TMD product could be an inexpensive, safe and convenient alternative for dysphagic patients [76,77]. Our referenced studies support the use of fortification in TMDs as a cost-effective approach to improving nutritional intake $[47,55]$. Similar findings have been seen in studies of hospital patients on regular diets where the use of food fortification has improved nutritional intake [78,79]. Considering the increasing use of TMDs, future studies should include cost effectiveness of fortification, thickeners, shaping powder and moulds used for shaping/moulding as references for food service.

The participants included in the review had various conditions, and included acute patients and chronic aged-care residents. Existing medical conditions may affect the assessment of nutritional status and responses of mealtime satisfaction. Future studies should consider analysing the association between patient aetiologies, clinical reasons related to TMD consumptions, nutritional status and mealtime satisfaction. The results may not be generalisable to individual patients with specific conditions. Evaluation of other clinical signs related to nutritional status and biochemistry makers, such as bowel movement, wound healing and blood test of micronutrient level should be considered in future studies, as very few studies incorporated these.

\section{Conclusions}

Poor nutritional status and meal acceptance was found in TMD and TF consumers. Although nutrition enrichment through fortification and modifying the texture and consistency of TMDs through shaping, moulding and consistency-control are promising strategies for improving nutritional status and preventing weight loss, there was a lack of evidence to make firm conclusions. The majority of the evidence used in this review was rated as neither strong nor weak. More high-quality research with follow-up and different interventions is required to generalise for larger populations. Future interventions should collaborate with food service to implement high-quality, nutritious TMDs, focusing on improving the nutrition content, appearance, flavour, taste, varieties and consistency. 
Supplementary Materials: The following are available online at https:/ / www.mdpi.com/article/10 .3390/healthcare9060624/s1, S1: Search strategy; S2: Prisma flow chart.

Author Contributions: Conceptualization, X.S.W. and A.J.B.; methodology, X.S.W., A.J.B. and A.M. database search, X.S.W.; data analysis, X.S.W. and A.J.B.; writing-original draft preparation, X.S.W.; writing-review and editing, A.M. and A.J.B. All authors have read and agreed to the published version of the manuscript.

Funding: This research received no external funding.

Institutional Review Board Statement: Not applicable.

Informed Consent Statement: Not applicable.

Data Availability Statement: The data used and/or analysed during the current study are available from the corresponding author on reasonable request.

Conflicts of Interest: The authors declare no conflict of interest.

\section{References}

1. Clavé, P.; Shaker, R. Dysphagia: Current reality and scope of the problem. Nat. Rev. Gastroenterol. Hepatol. 2015, 12, 259-270. [CrossRef]

2. Cichero, J.A.Y.; Steele, C.M.; Duivestein, J.; Clavé, P.; Chen, J.; Kayashita, J.; Dantas, R.D.O.; Lecko, C.; Speyer, R.; Lam, P.; et al. The Need for International Terminology and Definitions for Texture-Modified Foods and Thickened Liquids Used in Dysphagia Management: Foundations of a Global Initiative. Curr. Phys. Med. Rehabilit. Rep. 2013, 1, 280-291. [CrossRef] [PubMed]

3. Rothenberg, E.; Wendin, K. Texture Modification of Food for Elderly People; Elsevier BV: Amsterdam, The Netherlands, 2015; Volume 2, pp. 163-185.

4. Elmståhl, S.; Bülow, M.; Ekberg, O.; Petersson, M.; Tegner, H. Treatment of Dysphagia Improves Nutritional Conditions in Stroke Patients. Dysphagia 1999, 14, 61-66. [CrossRef] [PubMed]

5. Namasivayam, A.M.; Steele, C.M. Malnutrition and Dysphagia in Long-Term Care: A Systematic Review. J. Nutr. Gerontol. Geriatr. 2015, 34, 1-21. [CrossRef]

6. Peladic, N.J.; Orlandoni, P.; Dell'Aquila, G.; Carrieri, B.; Eusebi, P.; Landi, F.; Volpato, S.; Zuliani, G.; Lattanzio, F.; Cherubini, A. Dysphagia in Nursing Home Residents: Management and Outcomes. J. Am. Med. Dir. Assoc. 2019, 20, 147-151. [CrossRef]

7. Keller, H.; Chambers, L.; Niezgoda, H.; Duizer, L. Issues associated with the use of modified texture foods. J. Nutr. Health Aging 2011, 16, 195-200. [CrossRef]

8. Peñalva-Arigita, A.; Prats, R.; Lecha, M.; Sansano, A.; Vila, L. Prevalence of dysphagia in a regional hospital setting: Acute care hospital and a geriatric sociosanitary care hospital: A cross-sectional study. Clin. Nutr. ESPEN 2019, 33, 86-90. [CrossRef]

9. Tran, T.P.; Nguyen, L.T.; Kayashita, J.; Shimura, F.; Yamamoto, S. Nutritional Status and Feeding Practice among Dysphagic Older Adult Inpatients in Vietnam. J. Nutr. Sci. Vitaminol. 2020, 66, 224-228. [CrossRef] [PubMed]

10. O'Keeffe, M.; Kelly, M.; O’Herlihy, E.; O’Toole, P.; Kearney, P.; Timmons, S.; O'Shea, E.; Stanton, C.; Hickson, M.; Rolland, Y.; et al. Potentially modifiable determinants of malnutrition in older adults: A systematic review. Clin. Nutr. 2019, 38, $2477-2498$. [CrossRef] [PubMed]

11. Carrión, S.; Cabré, M.; Monteis, R.; Roca, M.; Palomera, E.; Serra-Prat, M.; Rofes, L.; Clavé, P. Oropharyngeal dysphagia is a prevalent risk factor for malnutrition in a cohort of older patients admitted with an acute disease to a general hospital. Clin. Nutr. 2015, 34, 436-442. [CrossRef] [PubMed]

12. Mann, T.; Heuberger, R.; Wong, H. The association between chewing and swallowing difficulties and nutritional status in older adults. Aust. Dent. J. 2013, 58, 200-206. [CrossRef]

13. Suominen, M.; Muurinen, S.; Routasalo, P.; Soini, H.; Suur-Uski, I.; Peiponen, A.; Finne-Soveri, H.; Pitkala, K.H. Malnutrition and associated factors among aged residents in all nursing homes in Helsinki. Eur. J. Clin. Nutr. 2005, 59, 578-583. [CrossRef] [PubMed]

14. Garcia, J.M.; Chambers, E. Managing Dysphagia Through Diet Modifications. Am. J. Nurs. 2010, 110, 26-33. [CrossRef]

15. Bannerman, E.; McDermott, K. Dietary and Fluid Intakes of Older Adults in Care Homes Requiring a Texture Modified Diet: The Role of Snacks. J. Am. Med. Dir. Assoc. 2011, 12, 234-239. [CrossRef] [PubMed]

16. Johnson, R.M.; Smiciklas-Wright, H.; Soucy, I.M.; Rizzo, J.A. Nutrient Intake of Nursing-Home Residents Receiving Pureed Foods or a Regular Diet. J. Am. Geriatr. Soc. 1995, 43, 344-348. [CrossRef]

17. Massoulard, A.; Bonnabau, H.; Gindre-Poulvelarie, L.; Baptistev, A.; Preux, P.-M.; Villemonteix, C.; Javerliat, V.; Fraysse, J.-L.; Desport, J.-C. Analysis of the food consumption of 87 elderly nursing home residents, depending on food texture. J. Nutr. Health Aging 2010, 15, 192-195. [CrossRef] [PubMed]

18. Painter, V.; Le Couteur, D.G.; Waite, L.M. Texture-modified food and fluids in dementia and residential aged care facilities. Clin. Interv. Aging 2017, 12, 1193-1203. [CrossRef] [PubMed] 
19. Wakabayashi, H.; Matsushima, M. Dysphagia assessed by the 10-item eating assessment tool is associated with nutritional status and activities of daily living in elderly individuals requiring long-term care. J. Nutr. Health Aging 2016, 20, 22-27. [CrossRef] [PubMed]

20. Serra-Prat, M.; Hinojosa, G.; López, D.; Juan, M.; Fabré, E.; Voss, D.S.; Calvo, M.; Marta, V.; Ribó, L.; Palomera, E.; et al. Prevalence of Oropharyngeal Dysphagia and Impaired Safety and Efficacy of Swallow in Independently Living Older Persons. J. Am. Geriatr. Soc. 2011, 59, 186-187. [CrossRef] [PubMed]

21. Shim, J.S.; Oh, B.-M.; Han, T.R. Factors Associated With Compliance With Viscosity-Modified Diet Among Dysphagic Patients. Ann. Rehabilit. Med. 2013, 37, 628-632. [CrossRef]

22. O'Keeffe, S.T. Use of modified diets to prevent aspiration in oropharyngeal dysphagia: Is current practice justified? BMC Geriatr. 2018, 18, 167. [CrossRef] [PubMed]

23. Karagiannis, M.J.; Chivers, L.; Karagiannis, T.C. Effects of oral intake of water in patients with oropharyngeal dysphagia. BMC Geriatr. 2011, 11, 9. [CrossRef] [PubMed]

24. Milne, A.C.; Potter, J.; Vivanti, A.; Avenell, A. Protein and energy supplementation in elderly people at risk from malnutrition. Cochrane Database Syst. Rev. 2009, 2009, CD003288. [CrossRef] [PubMed]

25. Collins, J.; Porter, J. The effect of interventions to prevent and treat malnutrition in patients admitted for rehabilitation: A systematic review with meta-analysis. J. Hum. Nutr. Diet. 2014, 28, 1-15. [CrossRef]

26. Divert, C.; Laghmaoui, R.; Crema, C.; Issanchou, S.; Van Wymelbeke, V.; Sulmont-Rossé, C. Improving meal context in nursing homes. Impact of four strategies on food intake and meal pleasure. Appetite 2015, 84, 139-147. [CrossRef]

27. Andersen, U.T.; Beck, A.M.; Kjaersgaard, A.; Hansen, T.; Poulsen, I. Systematic review and evidence based recommendations on texture modified foods and thickened fluids for adults ( $\geq 18$ years) with oropharyngeal dysphagia. e-SPEN J. 2013, 8, e127-e134. [CrossRef]

28. Steele, C.M.; Alsanei, W.A.; Ayanikalath, S.; Barbon, C.E.A.; Chen, J.; Cichero, J.A.Y.; Coutts, K.; Dantas, R.O.; Duivestein, J.; Giosa, L.; et al. The Influence of Food Texture and Liquid Consistency Modification on Swallowing Physiology and Function: A Systematic Review. Dysphagia 2015, 30, 2-26. [CrossRef]

29. Min, C.; Park, B.; Sim, S.; Choi, H.G. Dietary modification for laryngopharyngeal reflux: Systematic review. J. Laryngol. Otol. 2019, 133, 80-86. [CrossRef]

30. Foley, N.; Teasell, R.; Salter, K.; Kruger, E.; Martino, R. Dysphagia treatment post stroke: A systematic review of randomised controlled trials. Age Ageing 2008, 37, 258-264. [CrossRef] [PubMed]

31. Swan, K.; Speyer, R.; Heijnen, B.J.; Wagg, B.; Cordier, R. Living with oropharyngeal dysphagia: Effects of bolus modification on health-related quality of life-a systematic review. Qual. Life Res. 2015, 24, 2447-2456. [CrossRef] [PubMed]

32. Wu, X.; Miles, A.; Braakhuis, A. Nutritional Intake and Meal Composition of Patients Consuming Texture Modified Diets and Thickened Fluids: A Systematic Review and Meta-Analysis. Healthcare 2020, 8, 579. [CrossRef] [PubMed]

33. Sodeman, W.A.; Sodeman, T.C. Diets. In Instructions for Geriatric Patients; Elsevier BV: Amsterdam, The Netherlands, 2005; pp. 267-315.

34. Foeckler, P.; Henning, V.; Reichelt, J. Mendeley [Computer Program]; Version 1.19.4; Elsevier: London, UK, 2019; Available online: https: / / www.mendeley.com (accessed on 24 May 2021).

35. Microsoft Excel for Office 365 [Computer Program]; Version 1902; Microsoft Corp.: Redmond, WA, USA, 2019; Available online: https:/ / office.microsoft.com/ excel (accessed on 24 May 2021).

36. Wright, L.; Cotter, D.; Hickson, M.; Frost, G. Comparison of energy and protein intakes of older people consuming a texture modified diet with a normal hospital diet. J. Hum. Nutr. Diet. 2005, 18, 213-219. [CrossRef]

37. A Reyes-Torres, C.; Castillo-Martínez, L.; Reyes-Guerrero, R.; Ramos-Vázquez, A.G.; Zavala-Solares, M.; Cassis-Nosthas, L.; Serralde-Zúñiga, A.E. Design and implementation of modified-texture diet in older adults with oropharyngeal dysphagia: A randomized controlled trial. Eur. J. Clin. Nutr. 2019, 73, 989-996. [CrossRef]

38. Kaiser, M.J.; MNA-International Group; Bauer, J.M.; Ramsch, C.; Uter, W.; Guigoz, Y.; Cederholm, T.; Thomas, D.R.; Anthony, P.; Charlton, K.E.; et al. Validation of the Mini Nutritional Assessment short-form (MNA®-SF): A practical tool for identification of nutritional status. J. Nutr. Health Aging 2009, 13, 782-788. [CrossRef] [PubMed]

39. Shimizu, A.; Momosaki, R.; Kayashita, J.; Fujishima, I. Impact of Multiple Texture-Modified Diets on Oral Intake and Nutritional Status in Older Patients with Pneumonia: A Retrospective Cohort Study. Dysphagia 2019, 35, 574-582. [CrossRef] [PubMed]

40. Vellas, B.; Guigoz, Y.; Garry, P.J.; Nourhashemi, F.; Bennahum, D.; Lauque, S.; Albarede, J.-L. The mini nutritional assessment (MNA) and its use in grading the nutritional state of elderly patients. Nutrition 1999, 15, 116-122. [CrossRef]

41. Zanini, M.; Bagnasco, A.; Catania, G.; Aleo, G.; Sartini, M.; Cristina, M.L.; Ripamonti, S.; Monacelli, F.; Odetti, P.; Sasso, L. A Dedicated Nutritional Care Program (NUTRICARE) to reduce malnutrition in institutionalised dysphagic older people: A quasi-experimental study. J. Clin. Nurs. 2017, 26, 4446-4455. [CrossRef]

42. Martin, A.; Ortega, O.; Roca, M.; Arus, M.; Civit, P.C. Effect of a Minimal-Massive Intervention in Hospitalized Older Patients with Oropharyngeal Dysphagia: A Proof of Concept Study. J. Nutr. Health Aging 2018, 22, 739-747. [CrossRef]

43. Espinosa-Val, M.C.; Martín-Martínez, A.; Graupera, M.; Arias, O.; Elvira, A.; Cabré, M.; Palomera, E.; Bolívar-Prados, M.; Clavé, P.; Ortega, O. Prevalence, Risk Factors, and Complications of Oropharyngeal Dysphagia in Older Patients with Dementia. Nutrients 2020, 12, 863. [CrossRef] [PubMed] 
44. Miles, A.; Dennison, K.; Oad, M.A.; Shasha, L.; Royal, M. Consumer Satisfaction of Texture Modified Meals Served in Residential Aged-Care Facilities. Int. J. Food Sci. Nutr. Res. 2019, 1, 1. [CrossRef]

45. Vucea, V.; Keller, H.H.; Morrison, J.M.; Duizer, L.M.; Duncan, A.M.; Steele, C.M. Prevalence and Characteristics Associated with Modified Texture Food Use in Long Term Care: An Analysis of Making the Most of Mealtimes (M3) Project. Can. J. Diet. Pract. Res. 2019, 80, 104-110. [CrossRef]

46. Maeda, K.; Ishida, Y.; Nonogaki, T.; Shimizu, A.; Yamanaka, Y.; Matsuyama, R.; Kato, R.; Mori, N. Burden of Premorbid Consumption of Texture Modified Diets in Daily Life on Nutritional Status and Outcomes of Hospitalization. J. Nutr. Health Aging 2019, 23, 973-978. [CrossRef]

47. Cassens, D.; Johnson, E. Enhancing taste, texture, appearance and presentation of pureed foods improves resident quality of life and weight status. J. Am. Diet. Assoc. 1993, 93, A39. [CrossRef]

48. Farrer, O.; Olsen, C.; Mousley, K.; Teo, E. Does presentation of smooth pureed meals improve patients consumption in an acute care setting: A pilot study. Nutr. Diet. 2015, 73, 405-409. [CrossRef]

49. Garon, B.R.; Engle, M.; Ormiston, C. A Randomized Control Study to Determine the Effects of Unlimited Oral Intake of Water in Patients with Identified Aspiration. Neurorehabilit. Neural Repair 1997, 11, 139-148. [CrossRef]

50. Gellrich, N.-C.; Handschel, J.; Holtmann, H.; Krüskemper, G. Oral Cancer Malnutrition Impacts Weight and Quality of Life. Nutrients 2015, 7, 2145-2160. [CrossRef] [PubMed]

51. Germain, I.; Dufresne, T.; Gray-Donald, K. A Novel Dysphagia Diet Improves the Nutrient Intake of Institutionalized Elders. J. Am. Diet. Assoc. 2006, 106, 1614-1623. [CrossRef] [PubMed]

52. Higashiguchi, T. Novel diet for patients with impaired mastication evaluated by consumption rate, nutrition intake, and questionnaire. Nutrients 2013, 29, 858-864. [CrossRef] [PubMed]

53. Keller, H.H.; Chambers, L.W.; Fergusson, D.A.; Niezgoda, H.; Parent, M.; Caissie, D.; Lemire, N. A Mix of Bulk and Ready-to-Use Modified-Texture Food: Impact on Older Adults Requiring Dysphagic Food. Can. J. Aging 2012, 31, 335-348. [CrossRef] [PubMed]

54. Keller, H.H.; Duizer, L.M. What Do Consumers Think of Pureed Food? Making the Most of the Indistinguishable Food. J. Nutr. Gerontol. Geriatr. 2014, 33, 139-159. [CrossRef]

55. Kennewell, S.; Kokkinakos, M. Thick, cheap and easy: Fortifying texture-modified meals with infant cereal. Nutr. Diet. 2007, 64, 112-115. [CrossRef]

56. Konishi, M.; Kakimoto, N. Relationship between oral and nutritional status of older residents with severe dementia in an aged care nursing home. Gerodontology 2020. [CrossRef]

57. Okabe, Y.; Furuta, M.; Akifusa, S.; Takeuchi, K.; Adachi, M.; Kinoshita, T.; Kikutani, T.; Nakamura, S.; Yamashita, Y. Swallowing function and nutritional status in Japanese elderly people receiving home-care services: A 1-year longitudinal study. J. Nutr. Health Aging 2015, 20, 697-704. [CrossRef]

58. Ott, A.; Senger, M.; Lötzbeyer, T.; Gefeller, O.; Sieber, C.C.; Volkert, D. Effects of a Texture-Modified, Enriched, and Reshaped Diet on Dietary Intake and Body Weight of Nursing Home Residents with Chewing and/or Swallowing Problems: An Enable Study. J. Nutr. Gerontol. Geriatr. 2019, 38, 361-376. [CrossRef] [PubMed]

59. Shimizu, A.; Maeda, K.; Tanaka, K.; Ogawa, M.; Kayashita, J. Texture-modified diets are associated with decreased muscle mass in older adults admitted to a rehabilitation ward. Geriatr. Gerontol. Int. 2018, 18, 698-704. [CrossRef]

60. Welch, P.K.; Dowson, M.; Endres, J.M. The Effect of Nutrient Supplements on High Risk Long Term Care Residnets Receiving Pureed Diets. J. Nutr. Elder. 1991, 10, 49-62. [CrossRef]

61. Pritchard, S.J.; Davidson, I.; Jones, J.; Bannerman, E. A randomised trial of the impact of energy density and texture of a meal on food and energy intake, satiation, satiety, appetite and palatability responses in healthy adults. Clin. Nutr. 2014, 33, 768-775. [CrossRef]

62. Pauly, L.; Stehle, P.; Volkert, P.-D.D.D. Nutritional situation of elderly nursing home residents. Zeitschrift für Gerontologie und Geriatrie 2007, 40, 3-12. [CrossRef]

63. Popman, A.; Richter, M.; Allen, J.; Wham, C. High nutrition risk is associated with higher risk of dysphagia in advanced age adults newly admitted to hospital. Nutr. Diet. 2017, 75, 52-58. [CrossRef] [PubMed]

64. Ortega, O.; Martín, A.; Clavé, P. Diagnosis and Management of Oropharyngeal Dysphagia Among Older Persons, State of the Art. J. Am. Med. Dir. Assoc. 2017, 18, 576-582. [CrossRef]

65. Ueshima, J.; Momosaki, R.; Shimizu, A.; Motokawa, K.; Sonoi, M.; Shirai, Y.; Uno, C.; Kokura, Y.; Shimizu, M.; Nishiyama, A.; et al. Nutritional Assessment in Adult Patients with Dysphagia: A Scoping Review. Nutrients 2021, 13, 778. [CrossRef] [PubMed]

66. Yeh, S.-H.; Pan, M.-H. Chewing Screen and Interventions for Older Adults. Hu Li Za Zhi 2020, 67, 6-13. [CrossRef] [PubMed]

67. Smoliner, C.; Norman, K.; Scheufele, R.; Hartig, W.; Pirlich, M.; Lochs, H. Effects of food fortification on nutritional and functional status in frail elderly nursing home residents at risk of malnutrition. Nutrition 2008, 24, 1139-1144. [CrossRef]

68. Ballesteros-Pomar, M.D.; Cherubini, A.; Keller, H.; Lam, P.; Rolland, Y.; Simmons, S.F. Texture-Modified Diet for Improving the Management of Oropharyngeal Dysphagia in Nursing Home Residents: An Expert Review. J. Nutr. Health Aging 2020, 24, 576-581. [CrossRef] [PubMed]

69. Engh, M.C.N.; Speyer, R. Management of Dysphagia in Nursing Homes: A National Survey. Dysphagia 2021. [CrossRef]

70. Seemer, J.; Kiesswetter, E.; Fleckenstein, D.; Gloning, M.; Lötzbeyer, T.; Mittermaier, S.; Sieber, C.; Wurm, S.; Volkert, D. Effects of an individualised nutritional intervention on dietary intake and quality of life in nursing homes residents with (risk of) malnutrition: An enable study. Clin. Nutr. ESPEN 2020, 40, 681-682. [CrossRef] 
71. Bech, C.; Østergaard, T.; Knudsen, A.; Munk, T. An optimized texture modified diet improves energy and protein intake in hospitalized patients with dysphagia. Clin. Nutr. ESPEN 2020, 40, 514. [CrossRef]

72. MacQueen, C.E.; Taubert, S.; Cotter, D.; Stevens, S.; Frost, G.S. Which Commercial Thickening Agent Do Patients Prefer? Dysphagia 2003, 18, 46-52. [CrossRef]

73. Sungsinchai, S.; Niamnuy, C.; Wattanapan, P.; Charoenchaitrakool, M.; Devahastin, S. Texture Modification Technologies and Their Opportunities for the Production of Dysphagia Foods: A Review. Compr. Rev. Food Sci. Food Saf. 2019, 18, 1898-1912. [CrossRef]

74. Hickson, M.; Bulpitt, C.; Nunes, M.; Peters, R.; Cooke, J.; Nicholl, C.; Frost, G. Does additional feeding support provided by health care assistants improve nutritional status and outcome in acutely ill older in-patients? - a randomised control trial. Clin. Nutr. 2004, 23, 69-77. [CrossRef]

75. Liu, W.; Williams, K.; Batchelor-Murphy, M.; Perkhounkova, Y.; Hein, M. Eating performance in relation to intake of solid and liquid food in nursing home residents with dementia: A secondary behavioral analysis of mealtime videos. Int. J. Nurs. Stud. 2019, 96, 18-26. [CrossRef] [PubMed]

76. Ballesteros-Pomar, M.; Perez-Martin, J.; Mendiola, J.; Garcia-Garcia, J.; Parrado, S.; Caracuel, A.; Garces, B.; de Paz, H.; Bar-cons, N. Energy intake and effects on swallowing function of institutionalized elderly consuming a texture-modified diets in three types of production, ABADIA study. Eur. Geriatr. Med. 2019, 10, 1-325. [CrossRef]

77. Ballesteros, M.; Pérez-Martín, J.; Mendiola, M.; García-García, J.; Parrado, S.; Caracuel, A.; Garcés, B.; Serra, M.C.; Barcons, N. Cost and nutritional value of three alternative texture-modified diets for patients with swallowing or chewing disorders: ABADIA study. Value Health 2019, 22, S416. [CrossRef]

78. Walton, K.; Williams, P.; Tapsell, L. Improving food services for elderly, long-stay patients in Australian hospitals: Adding food fortification, assistance with packaging and feeding assistance. Nutr. Diet. 2012, 69, 137-144. [CrossRef]

79. Gall, M.; Grimble, G.; Reeve, N.; Thomas, S. Effect of providing fortified meals and between-meal snacks on energy and protein intake of hospital patients. Clin. Nutr. 1998, 17, 259-264. [CrossRef] 